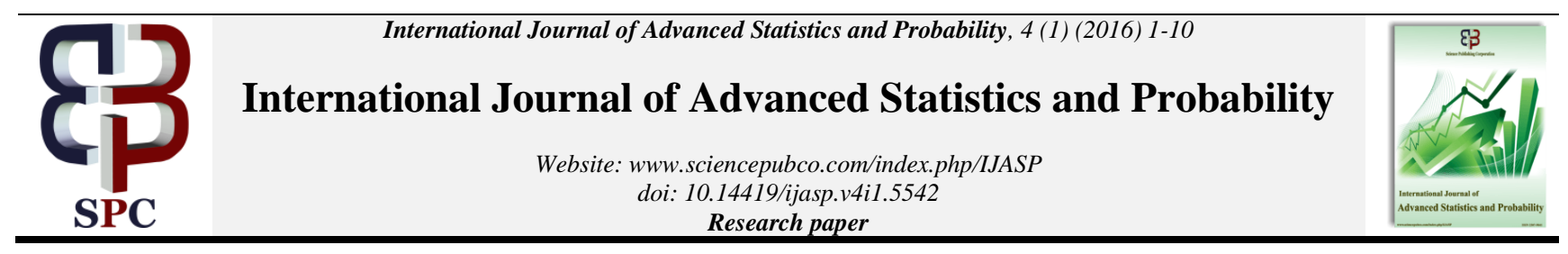

\title{
Bayesian estimation of the shape parameter of generalized Rayleigh distribution under non-informative prior
}

\author{
AliyuYakubu*, AbubakarYahaya \\ Department of Mathematics, Ahmadu Bello University, Zaria-Nigeria \\ *Corresponding author E-mail:aliyuyakubu40@yahoo.com
}

\begin{abstract}
A decade ago, two-parameter Burr Type X distribution was introduced by Surles and Padgett [14] which was described as Generalized Rayleigh Distribution (GRD). This skewed distribution can be used quiet effectively in modelling life time data. In this work, Bayesian estimation of the shape parameter of GRD was considered under the assumption of non-informative prior. The estimates were obtained under the squared error, Entropy and Precautionary loss functions. Extensive Monte Carlo simulations were carried out to compare the performances of the Bayes estimates with that of MLEs. It was observed that the estimate under the Entropy loss function is more stable than the estimates under squared error loss function, Precautionary loss function and MLEs.
\end{abstract}

Keywords: Bayes Estimators; Extended Jeffrey's Prior; Entropy Loss Function; Precautionary Loss Function; Squared Error Loss Function.

\section{Introduction}

Twelve different families of cummulative distribution function used in modelling lifetime data was suggested by Burr [3]. Burr Type $\mathrm{X}$ distribution is among the most popular distributions that receives the most attention among these families of cummulative distributions. In 2001, two parameter Burr Type X distribution was introduced by Surles and Padgett [14]. Abdel-Hady [1], Kundu and Raqab [8] and Lio, Chen and Tsai [9] prefer to call this distribution GRD; this name will be adopted in this work. For $\alpha>0$ and $\lambda>0$, the two-parameter GRD will be denoted by $\operatorname{GRD}(\alpha, \lambda)$ and its cumulative distribution function is given by:

$\mathrm{F}(\mathrm{x} ; \alpha, \lambda)=\left(1-\mathrm{e}^{-(\lambda \mathrm{x})^{2}}\right)^{\alpha} \quad$ for $\mathrm{x}, \alpha, \lambda>0$

Its probability density function (pdf) is given by:

$F(x ; \alpha, \lambda)=2 \alpha \lambda^{2} x\left(1-e^{-(\lambda x)^{2}}\right)^{\alpha-1} e^{-(\lambda x)^{2}}$

where $\alpha$ and $\lambda$ are the shape and scale parameters respectively.

The survival as well as the hazard functions are respectively given by:

$\mathrm{S}(\mathrm{x} ; \alpha, \lambda)=1-\mathrm{F}(\mathrm{x} ; \alpha, \lambda)=1-\left(1-\mathrm{e}^{-(\lambda \mathrm{x})^{2}}\right)^{\alpha}$

$\mathrm{h}(\mathrm{x} ; \alpha, \lambda)=\frac{\mathrm{f}(\mathrm{x} ; \alpha, \lambda)}{\mathrm{S}(\mathrm{x} ; \alpha, \lambda)}=\frac{2 \alpha \lambda^{2} \mathrm{x}\left(1-\mathrm{e}^{-(\lambda \mathrm{x})^{2}}\right)^{\alpha-1} \mathrm{e}^{-(\lambda \mathrm{x})^{2}}}{1-\left(1-\mathrm{e}^{-(\lambda x)^{2}}\right)^{\alpha}}$

GRD is widely used in modelling events that occur in different fields such as medicine, social and natural sciences. In Physics for instance, the GRD is used in the study of various types of radiations such as light and sound measurements. It is used as a model for wind speed and is often applied to wind driven electrical gen- eration. It can also be used in modelling strength and lifetime data (we refer an interested reader to, Kundu \& Raqab [8], Lio, Chen and Tsai [9], Samaila and Cenac [13] and Surles and Padgett [14]). The graph of the GRD is shown in figure 1 for different shape parameter values. It is clear from figure 1 that, the pdf of GRD is a decreasing function if $\alpha \leq 1 / 2$ and it is right skewed uni-modal when $\alpha>1 / 2$ see also Kundu and Raqab [8].

Kundu \& Raqab [8] used the methods of maximum likelihood, modified moment estimator, least squares estimator, weighted least squares estimator, percentile-based estimator and modified L-moment estimator to estimate the unknown parameters of GRD. The study compared the performances of the different estimators using Monte-Carlo simulations mainly with respect to their biases and mean squared errors (MSE) for different sample sizes and different parameter values. It was further shown that when the sample size is small (say $n=10$ ), the performances of most of the methods are quite discouraging. In particular, the estimation of $\alpha$ becomes very difficult for small sample sizes. The biases of all the methods are quite severe for small and moderate sample sizes (say $\mathrm{n} \leq 20$ ). It was also shown that the least square estimate (LSE) performs quite well for $n \leq 20$ and for $n \geq 30$, the weighted LSE outperforms the LSE marginally. If MSE is considered, the weighted LSE performs better than the rest in most of the cases considered. Also in Raqab and Kundu [12], a comparison was made on different methods of estimating $\mathrm{R}=\mathrm{P}(\mathrm{Y}<\mathrm{X})$ when $\mathrm{Y}$ and $\mathrm{X}$ both follow GRD with different shape parameters but the same scale parameter (ie. $\mathrm{X} \sim \operatorname{GRD}\left(\alpha_{1}, \lambda\right)$ and $\mathrm{Y} \sim \operatorname{GRD}\left(\alpha_{2}, \lambda\right)$ ). When the scale parameter is unknown, it is observed that the MLEs of the three unknown parameters can be obtained by solving one non-linear equation. An iterative procedure for computing the MLEs of the unknown parameters and the MLE of R was developed. The asymptotic distribution of $\mathrm{R}$ was obtained and this was used to compute the asymptotic confidence intervals. It is observed that even when the sample size is quite small the asymptotic confidence intervals work quite well. Two bootstrap confidence intervals were also proposed and their performances were 
also quite satisfactory. On the other hand, when the scale parameter is known they compared MLE and UMVUE with different Bayes estimators. It is observed that the Bayes estimators with non-informative priors behave in similar fashion as the MLEs

Mahdi [10] estimate the parameters of a Rayleigh distribution using five point estimation techniques; maximum likelihood estimates, moment method, probability weighted moment method, least square method and least absolute deviation method. The researcher also proposed the modified maximum likelihood estimation method for the parameters and compared it with the aforementioned methods. It was observed that, all the methods performed reasonably well except the method of moments. On the other hand, the modified maximum likelihood method provides better estimates for the parameters when the sample sizes are not small (say $n \geq 10$ ), while in the case of small samples, the probability weighted moment method outperforms the maximum likelihood method for the estimation of the threshold parameter and performs almost as good as the maximum likelihood method for the estimation of the scale parameter. Hence, Mahdi [10] recommends using the modified maximum likelihood method for the parameter estimation of the Rayleigh distribution if the sample size is large.

The rest of the paper is organized as follows: in section two, we briefly describe the MLE. In section 3 we describe the Bayesian method, Numerical comparisons are presented in section 4 and we finally conclude in section 5 .

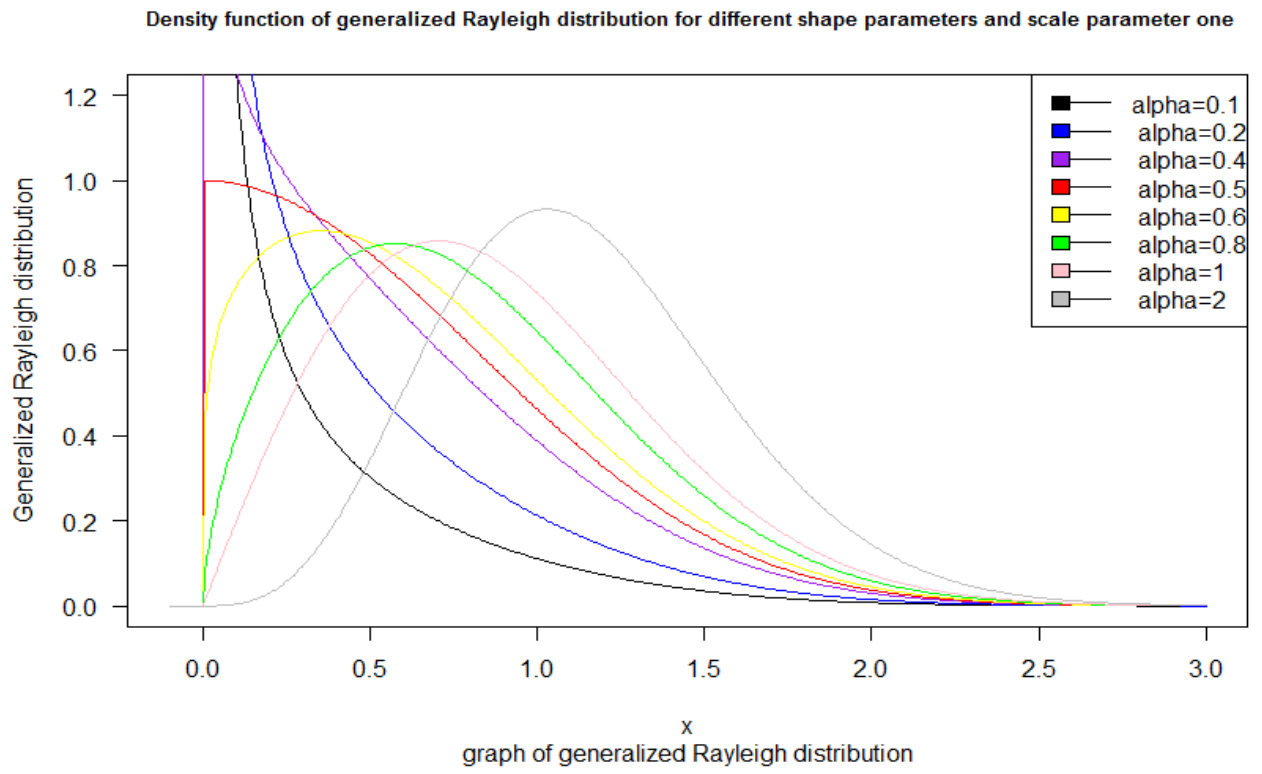

Fig.1: The Graph of Generalized Rayleigh Distribution for Different Values of Shape Parameters When the Scale Parameter Takes the Value One

\section{Maximum likelihood estimation}

Let $\mathrm{x}_{1}, \mathrm{x}_{2}, \cdots, \mathrm{x}_{\mathrm{n}}$ be a random sample from a population $\mathrm{X}$ with pdf $\mathrm{f}(\mathrm{x} ; \theta)$, where $\theta$ is an unknown parameter. The likelihood function, $\mathrm{L}(\theta)$, is defined to be the joint density of the random variables $\mathrm{x}_{1}, \mathrm{x}_{2}, \cdots, \mathrm{x}_{\mathrm{n}}$.

That is,

$$
L(\theta)=\prod_{i=1}^{n} f\left(x_{i} ; \theta\right)
$$

The value of the statistic that maximizes the likelihood function, $\mathrm{L}(\theta)$ is called the maximum likelihood estimator of $\theta$ and it is denoted by $\theta$. If the scale parameter $\lambda$ is known and without loss of generality let $\lambda=1$, then the pdf of $\operatorname{GRD}(\alpha, 1)$ is given by:

$f(x ; \alpha, 1)=2 \alpha x\left(1-e^{-x^{2}}\right)^{\alpha-1} e^{-x^{2}}$

and the likelihood of (5) is given by:

$L\left(x_{1}, x_{2}, \cdots, x_{n} ; \alpha, 1\right)=2^{n} \alpha^{n} e^{-\sum_{i=1}^{n} x_{i}^{2}} \prod_{i=1}^{n} x_{i}\left(1-e^{-x_{i}^{2}}\right)^{\alpha-1}$

Differentiating (6) with respect to $\alpha$, equating to zero and solving for $\alpha$ yeilds the MLE of $\alpha$ which will be denoted by $\widehat{\alpha}_{\text {MLE }}$. Hence, $\widehat{\alpha}_{M L E}$ is given by:

$$
\widehat{\alpha}_{\mathrm{MLE}}=-\frac{\mathrm{n}}{\sum_{\mathrm{i}=1}^{\mathrm{n}} \ln \left(1-\mathrm{e}^{-\mathrm{x}_{\mathrm{i}}^{2}}\right)}
$$

and its variance is obtained as

$\operatorname{var}\left(\widehat{\alpha}_{\mathrm{MLE}}\right)=\frac{\mathrm{n}^{2}}{(\mathrm{n}-1)^{2}(\mathrm{n}-2)} \widehat{\alpha}_{\mathrm{MLE}}^{2}$

\section{Bayesian estimation of the shape parameter}

Bayesian estimation technique will be used to estimate the shape parameter of the GRD assuming Extended Jeffrey's prior under the squared error loss function which is classified as symmetric loss function as well as Entropy and precautionary loss functions which are classified as asymmetric loss function.

\subsection{Squared error loss function}

The squared error loss function is defined as

$\mathrm{L}(\theta, \hat{\theta})=(\hat{\theta}-\theta)^{2}$

The Bayes estimator of $\theta$ denoted by $\hat{\theta}_{\mathrm{SL}}$ relative to the squared error loss function is given by

$\hat{\theta}_{\mathrm{SL}}=\mathrm{E}(\theta / \mathrm{x})$

The squared error loss function is classified as symmetric loss function, because it assigns equal importance to the losses due to overestimation and underestimation. This is impractical in real life 
situations since overestimation of a parameter can lead to more or less severe consequences than underestimation and vice versa as in the case of estimation of reliability or failure rate function. Hence, the loss function which associate greater importance to overestimation or underestimation and serves as alternative to symmetric loss function is the asymmetric loss function. Several asymmetric loss functions such as Entropy loss function, Precautionary loss function, LINEX loss function, weighted loss function, etc have been proposed and studied by many researchers. This paper will consider only Entropy loss function and Precautionary loss function

\subsection{Entropy loss function}

The Entropy loss function discussed by Dey [5] and Dey and Liu [6] is defined as:

$\mathrm{L}_{\mathrm{EL}}(\hat{\theta}, \theta) \propto\left(\frac{\widehat{\theta}}{\theta}\right)^{\mathrm{k}}-\mathrm{klog}\left(\frac{\widehat{\theta}}{\theta}\right)-1 \mathrm{k} \neq 0$

where $\hat{\theta}$ is an estimate of $\theta$.

The Bayes estimator of $\theta$ denoted by $\hat{\theta}_{\mathrm{EL}}$ relative to the entropy loss function is given by:

$\hat{\theta}_{\mathrm{EL}}=\left[\mathrm{E}_{\theta}\left(\theta^{-\mathrm{k}} / \mathrm{x}\right)\right]^{-\frac{1}{\mathrm{k}}}$

Provided $\mathrm{E}_{\theta}\left(\theta^{-\mathrm{k}} / \mathrm{x}\right)$ exist. If $\mathrm{k}=-1$, the bayes estimator (12) coincides with the bayes estimator under the squared error loss function and for $\mathrm{k}=1$, it coincides with the weighted squared error loss function.

\subsection{Precautionary loss function}

Precautionary loss function is defined by Norstrom [11] as:

$\mathrm{L}_{\mathrm{PL}}(\hat{\theta}, \theta)=\frac{(\widehat{\theta}-\theta)^{2}}{\widehat{\theta}}$

The Bayes estimator of $\theta$ denoted by $\hat{\theta}_{\mathrm{PL}}$ relative to the precautionary error loss function is given by:

$\hat{\theta}_{P L}=\left(E\left(\theta^{2} / x\right)\right)^{\frac{1}{2}}$

\subsection{Bayes estimation of the shape parameter under the extended Jeffrey's prior}

In this section, the bayes estimation of the unknown parameter of the $\operatorname{GRD}(\alpha, 1)$ assuming Extended Jeffrey's prior is obtained. Jeffrey [7] proposed a method for generating non-informative priors which is based on the Fisher Information Matrix given by:

$I(\alpha)=-E\left(\frac{\partial^{2} \ln f(X ; \alpha)}{\partial \alpha_{i} \partial \alpha_{j}}\right)$

The Jeffrey's prior is defined by:

$\pi(\alpha) \propto \sqrt{I(\alpha)}$

Al-Kutubi [2] proposed an extension to Jeffrey's prior which is defined as:

$\pi(\alpha) \propto(I(\alpha))^{r}, r \in \mathbb{R}^{+}$

where $I(\alpha)$ is as defined in equation (15)

In other to obtained $I(\alpha)$, we differentiate equation (6) twice with respect to $\alpha$ resulting in: $\frac{\partial^{2} \ln f(X ; \alpha)}{\partial \alpha^{2}}=-\frac{1}{\alpha^{2}}$

and hence the fisher information $I(\alpha)=\frac{1}{\alpha^{2}}$

Hence, from equation (17), it is easy to conclude that the extended Jeffrey's prior is defined as:

$\pi(\alpha)=\frac{1}{\alpha^{r}}$

Let's assume the shape parameter $\alpha$ is a random variable having Extended Jeffrey's prior given by (19) and let $x_{1}, x_{2}, \cdots, x_{n}$ be a random sample from $\operatorname{GRD}(\alpha, 1)$, then the likelihood of the observed data is given in (6), the product of the likelihood and the prior is given by:

$L\left(x_{1}, x_{2}, \cdots, x_{n} ; \alpha, 1\right) \pi(\alpha)=2^{n} \alpha^{n-r} e^{-\sum_{i=1}^{n} x_{i}^{2}} \prod_{i=1}^{n} x_{i}\left(1-e^{-x_{i}^{2}}\right)^{\alpha-1}$

while the posterior distribution of $\alpha$ is given by:

$$
\pi\left(\alpha / x_{1}, x_{2}, \cdots, x_{n}\right)=\frac{L\left(x_{1}, x_{2}, \cdots, x_{n} ; \alpha, 1\right) \pi(\alpha)}{\int_{0}^{\infty} L\left(x_{1}, x_{2}, \cdots, x_{n} ; \alpha, 1\right) \pi(\alpha) d \alpha}
$$

Integrating (20) with respect to $\alpha$, yeilds:

$\int_{0}^{\infty} L\left(x_{1}, x_{2}, \cdots, x_{n} ; \alpha, 1\right) \pi(\alpha) d \alpha=$
$\frac{2^{n} e^{-\sum_{i=1}^{n} x_{i}^{2}} \prod_{i=1}^{n} x_{i}\left(1-e^{-x_{i}^{2}}\right)^{-1}}{\left(\sum_{i=1}^{n} \ell n\left(1-e^{-x_{i}^{2}}\right)^{-1}\right)^{n-r+1}} \sqrt{n-r+1}$

Hence, the posterior distribution (21) becomes:

$\pi\left(\alpha / x_{1}, x_{2}, \cdots, x_{n}\right)=\frac{\alpha^{\mathrm{n}-\mathrm{r}} M^{n-r+1} e^{-M \alpha}}{\sqrt{n-r+1}}$

which is gamma distributed with parameters $(n-r+1)$ and $M$.

where $M=\sum_{i=1}^{n} \ln \left(1-e^{-x_{i}^{2}}\right)^{-1}$

Therefore, the Bayesian estimators of the parameter $\alpha$ under the Squared error, Entropy and Precautionary loss functions denoted by $\alpha_{S L}, \alpha_{E L}$ and $\alpha_{P L}$ are respectively given by (24), (25) and (26)

$\alpha_{S L}=\frac{n-r+1}{M}$

$\alpha_{E L}=\frac{1}{M}\left(\frac{\sqrt{n-r+1}}{\sqrt{n-r-k+1}}\right)^{\frac{1}{k}}$

$\alpha_{P L}=\frac{1}{M} \sqrt{(n-r+2)(n-r+1)}$

where $M=\sum_{i=1}^{n} \ell \mathrm{n}\left(1-e^{-x_{i}^{2}}\right)^{-1}$

\subsubsection{Variance}

If $M=\sum_{i=1}^{n} \ell \mathrm{n}\left(1-e^{-x_{i}^{2}}\right)^{-1}$, and $\mathrm{X} \sim G R D(\alpha, 1)$, it is easy to show that $M \sim \operatorname{Gamma}(n, \alpha)$ that is 
$f(m)=\frac{\alpha^{n} m^{n-1} e^{-\alpha m}}{\Gamma_{n}}$

and

$E\left(m^{g}\right)=\frac{\sqrt{n+g}}{\alpha^{g} \Gamma_{n}}$

Hence, the variance of the shape parameter under the aforementioned loss functions are given by:

$\operatorname{var}\left(\alpha_{S L}\right)=\frac{(n-r+1)^{2}}{(n-1)^{2}(n-2)} \alpha^{2}$

$\operatorname{var}\left(\alpha_{E L}\right)=\frac{\alpha^{2}}{(n-1)^{2}(n-2)}\left(\frac{\sqrt{n-r+1}}{\sqrt{n-r-k+1}}\right)^{\frac{2}{k}}$

$\operatorname{Var}\left(\alpha_{P L}\right)=\frac{(n-r+2)(n-r+1)}{(n-1)^{2}(n-2)} \alpha^{2}$

\subsubsection{Relative efficiency}

Various statistical properties of point estimators are used to decide which estimator is most appropriate, will give the least risk and the most information at the lowest cost. Relative Efficiency (R.E) is one of these desirable properties. In this section, the relative efficiency of the proposed estimators will be obtained.

The relative efficiency of $\alpha_{E L}$ with respect to $\alpha_{S L}$ of $\alpha$ is

$E_{1}=\frac{\operatorname{Var}\left(\alpha_{S L}\right)}{\operatorname{Var}\left(\alpha_{E L}\right)}=(n-r+1)^{2}\left(\frac{\sqrt{n-r-k+1}}{\sqrt{n-r+1}}\right)^{\frac{2}{k}}$

$E_{1}>1$ for $k \geq 1, E_{1}=1$ for $k=-1$ and $E_{1}<1$ for $k<-1$ (see fig. $2 \&$ 3)

The relative efficiency of $\alpha_{E L}$ with respect to $\alpha_{P L}$ of $\alpha$ is
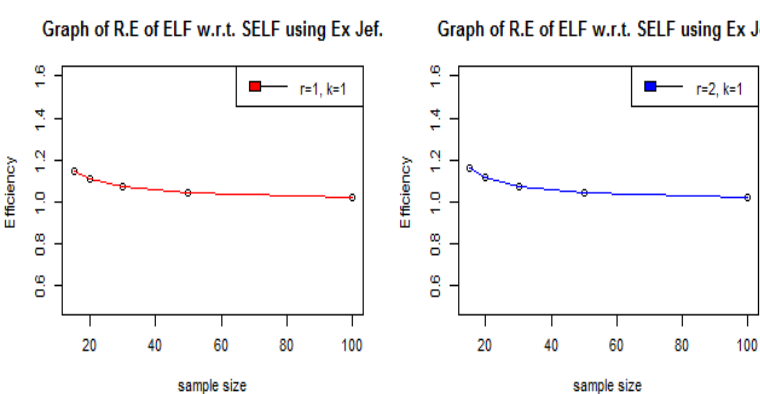

Graph of R.E of ELF w.r.t. SELF using Ex Jef.

Graph of R.E of ELF w.r.t. SELF using Ex Jef.
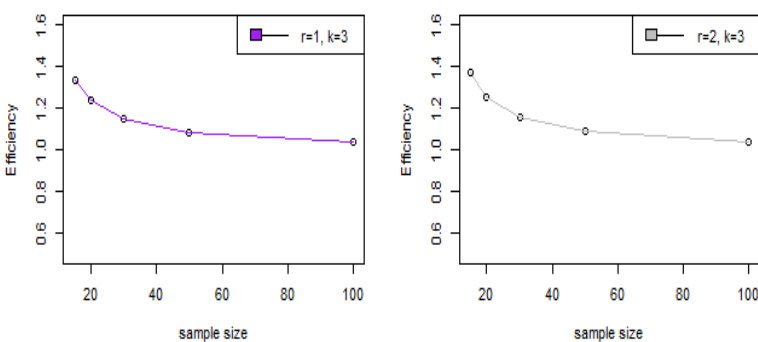

Fig. 2: Graph of Relative Efficiency of Entropy Loss Function with Respect to Squared Error Loss Function under Extended Jeffrey's Prior for Different Values of $\mathrm{k}=1,2,3,4$ and $\mathrm{r}=1,2$ when $\mathrm{n}=15,20,30,50$ and 100
$E_{2}=\frac{\operatorname{Var}\left(\alpha_{P L}\right)}{\operatorname{Var}\left(\alpha_{E L}\right)}=(n-r+2)(n-r+1)\left(\frac{\sqrt{n-r-k+1}}{\sqrt{n-r+1}}\right)^{\frac{2}{k}}$

$E_{2}>1$ for $k \geq-1, E_{2}=1$ for $k=-2$ and $E_{2}<1$ for $k<-2$ (see fig. $4 \& 5)$

The relative efficiency of $\alpha_{S L}$ with respect to $\alpha_{P L}$ of $\alpha$ is

$E_{3}=\frac{\operatorname{Var}\left(\alpha_{P L}\right)}{\operatorname{Var}\left(\alpha_{S L}\right)}=\frac{(n-r+2)}{(n-r+1)}$

$E_{3}>1$ when $r \geq 1$ (see fig. 8)

The relative efficiency of $\alpha_{S L}$ with respect to $\alpha_{M L E}$ of $\alpha$ is

$E_{4}=\frac{\operatorname{Var}\left(\alpha_{M L E}\right)}{\operatorname{Var}\left(\alpha_{S L}\right)}=\frac{n^{2}}{(n-r+1)^{2}}$

$E_{4}>1$ when $r>1$ (see fig. 8)

The relative efficiency of $\alpha_{E L}$ with respect to $\alpha_{M L E}$ of $\alpha$ is

$E_{5}=\frac{\operatorname{Var}\left(\alpha_{M L E}\right)}{\operatorname{Var}\left(\alpha_{E L}\right)}=n^{2}\left(\frac{\sqrt{n-r-k+1}}{\sqrt{n-r+1}}\right)^{\frac{2}{k}}$

$E_{5}>1$ when $k \geq 1$ (see fig. $6 \& 7$ )

The relative efficiency of $\alpha_{P L}$ with respect to $\alpha_{M L E}$ of $\alpha$ is

$E_{6}=\frac{\operatorname{Var}\left(\alpha_{M L E}\right)}{\operatorname{Var}\left(\alpha_{P L}\right)}=\frac{n^{2}}{(n-r+2)(n-r+1)}$

$E_{6}>1$ when $r \geq 2$ (see fig. 8)

Graph of R.E of ELF w.r.t. SELF using Ex Jef. Graph of R.E of ELF w.r.t. SELF using Ex Jef.
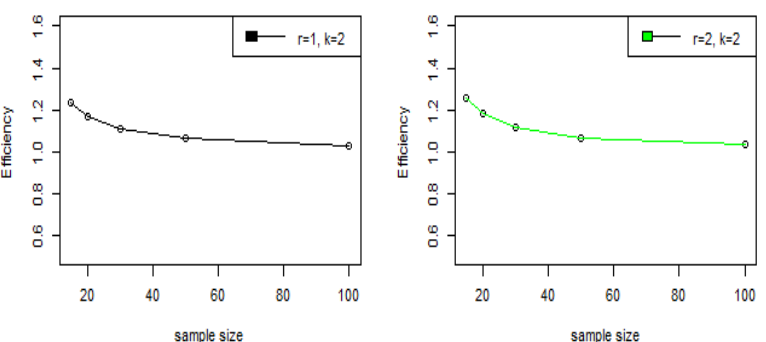

Graph of R.E of ELF w.r.t. SELF using Ex Jef.

Graph of R.E of ELF w.r.t. SELF using Ex Jef.
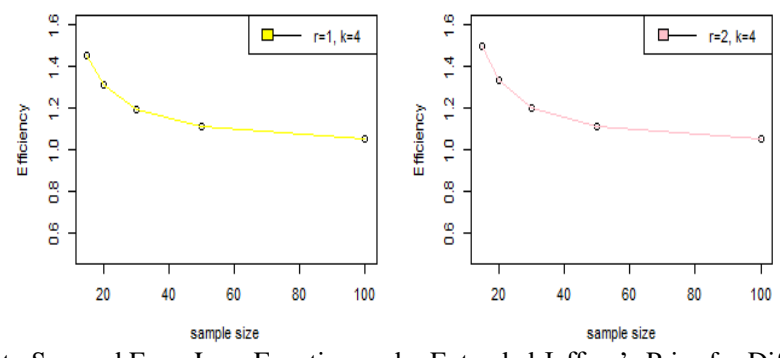

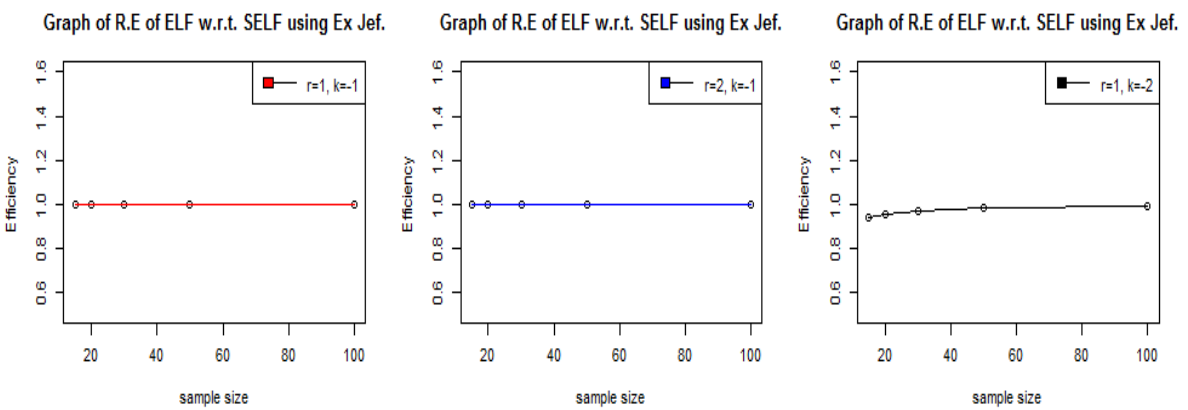

Graph of R.E of ELF w.r.t. SELF using Ex Jef.
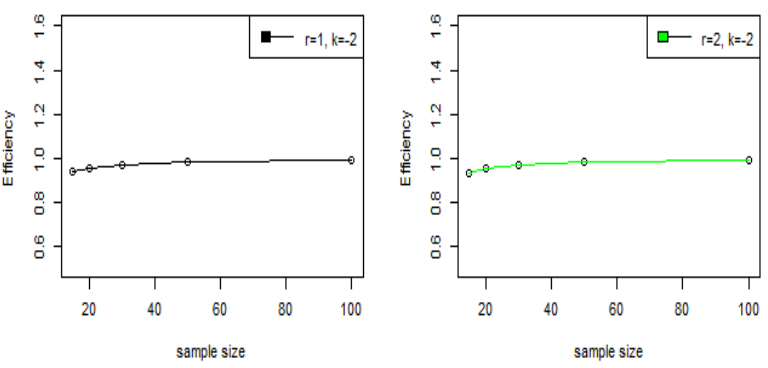

Graph of R.E of ELF w.r.t. SELF using Ex Jef.

Graph of R.E of ELF w.r.t. SELF using Ex Jef.

Graph of R.E of ELF w.r.t. SELF using Ex Jef.

Graph of R.E of ELF w.r.t. SELF using Ex Jef.
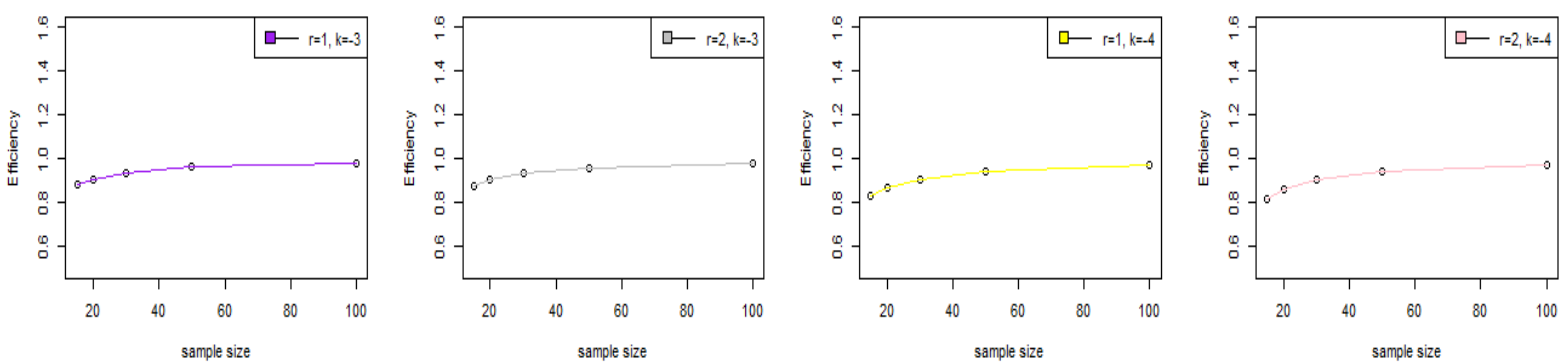

Fig. 3: Graph of Relative Efficiency of Entropy Loss Function With respect to Squared Error Loss Function under Extended Jeffrey's Prior for Different Values of $\mathrm{k}=-1,-2,-3,-4$ and $\mathrm{r}=1,2$ when $\mathrm{n}=15,20,30,50$ and 100

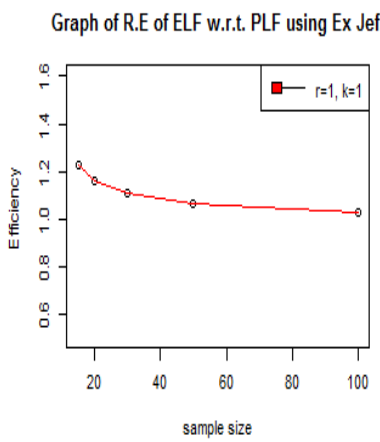

Graph of R.E of ELF w.r.t. PLF using Ex Jef.

Graph of R.E of ELF w.r.t. PLF using Ex Jef.

Graph of R.E of ELF w.r.t. PLF using Ex Jef.
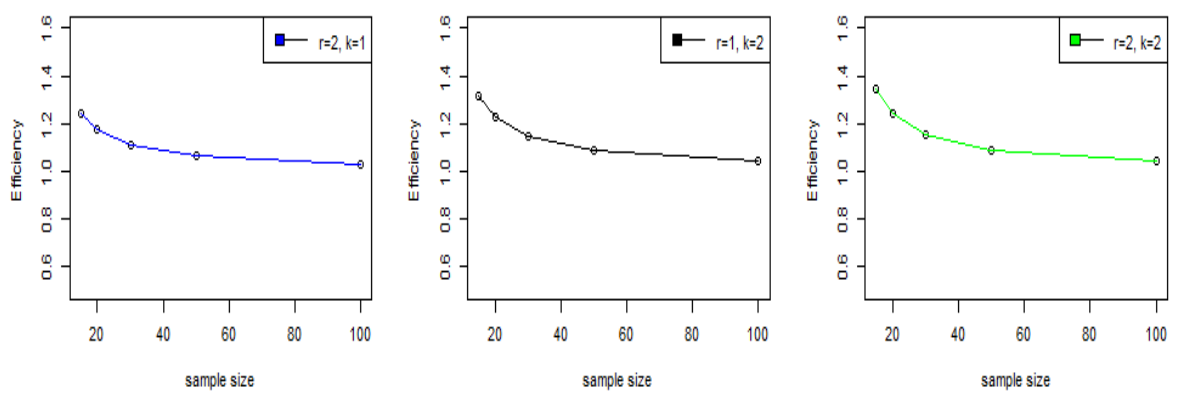

Graph of R.E of ELF w.r.t. PLF using Ex Jef.

Graph of R.E of ELF w.r.t. PLF using Ex Jef.

Graph of R.E of ELF w.r.t. PLF using Ex Jef.

Graph of R.E of ELF w.r.t. PLF using Ex Jef.
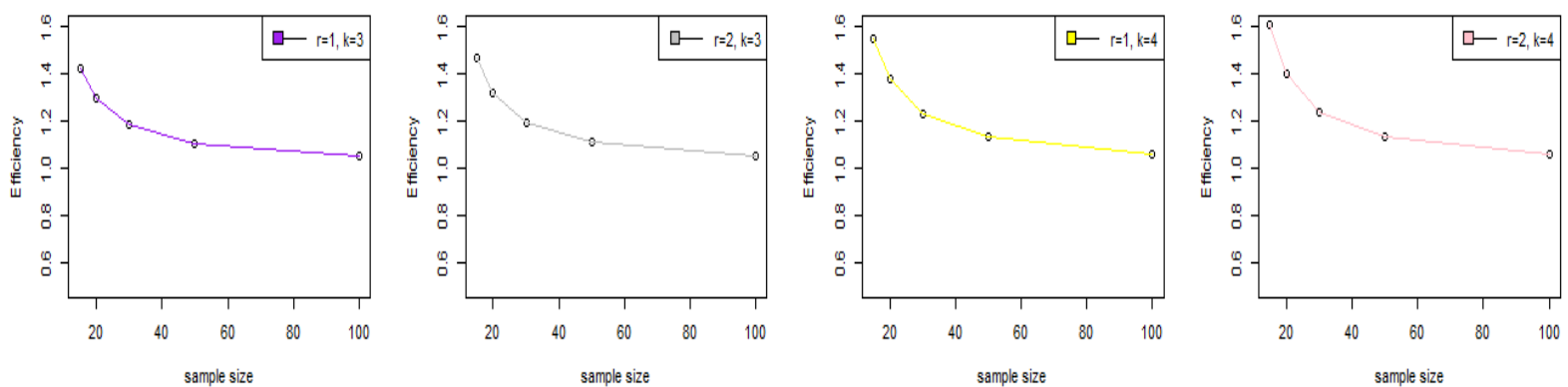

Fig. 4: Graph of Relative Efficiency of Entropy Loss Function with Respect to Precautionary Loss Function under Extended Jeffrey's Prior for Different Values of $\mathrm{k}=1,2,3,4$ and $\mathrm{r}=1,2$ when $\mathrm{n}=15,20,30,50$ and 100 
Graph of R.E of ELF w.r.t. PLF using Ex Jef.
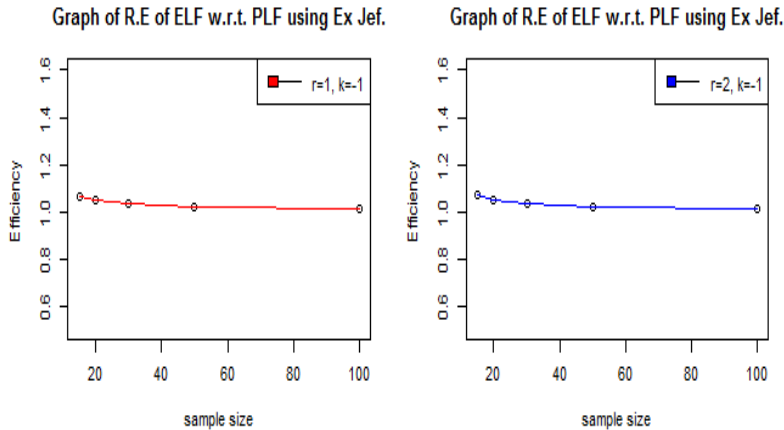

Graph of R.E of ELF w.r.t. PLF using Ex Jef.
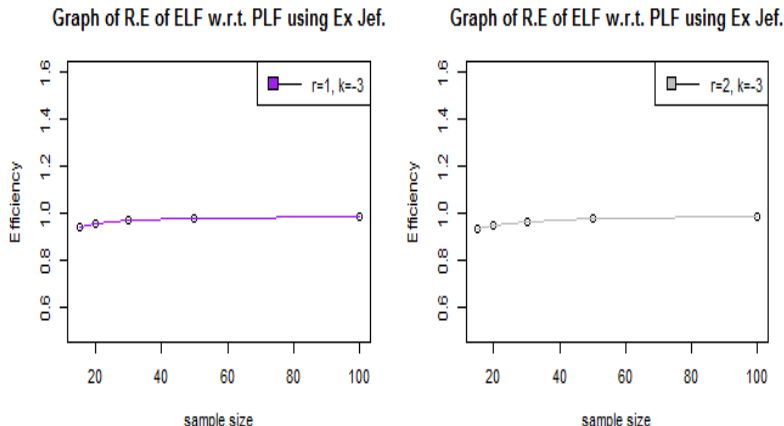

Graph of R.E of ELF w.r.t. PLF using Ex Jef.

Graph of R.E of ELF w.r.t. PLF using Ex Jef.
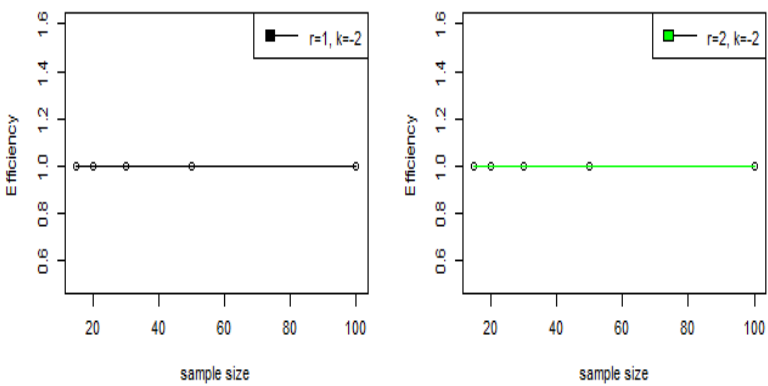

Graph of R.E of ELF w.r.t. PLF using Ex Jef.

Graph of R.E of ELF w.r.t. PLF using Ex Jef.

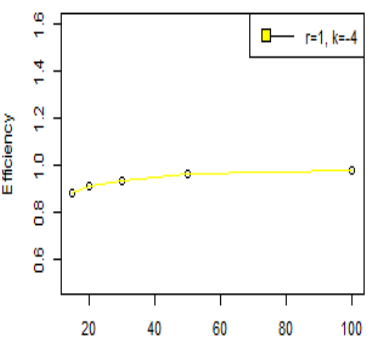

sample size

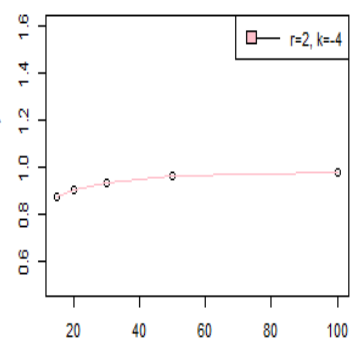

Fig. 5: Graph of Relative Efficiency of Entropy Loss Function with Respect to Precautionary Loss Function under Extended Jeffrey's Prior for Different Values of $\mathrm{k}=-1,-2,-3,-4$ and $\mathrm{r}=1,2$ when $\mathrm{n}=15,20,30,50$ and 100
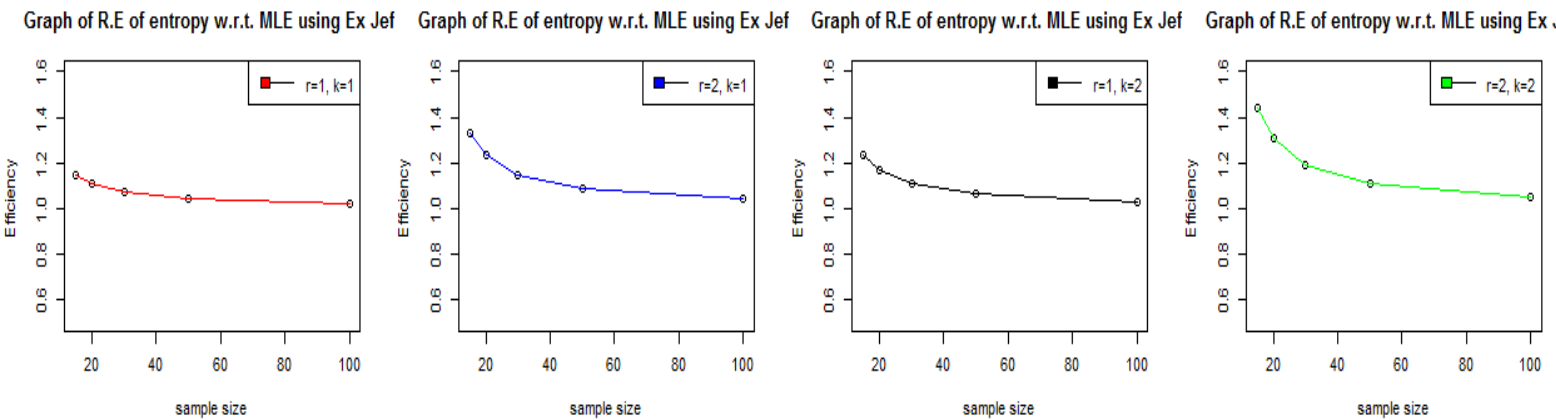

Graph of R.E of entropy w.r.t. MLE using Ex Jef Graph of R.E of entropy w.r.t. MLE using Ex Jef Graph of R.E of entropy w.r.t. MLE using Ex Je

Graph of R.E of entropy w.r.t. MLE using Ex Jel
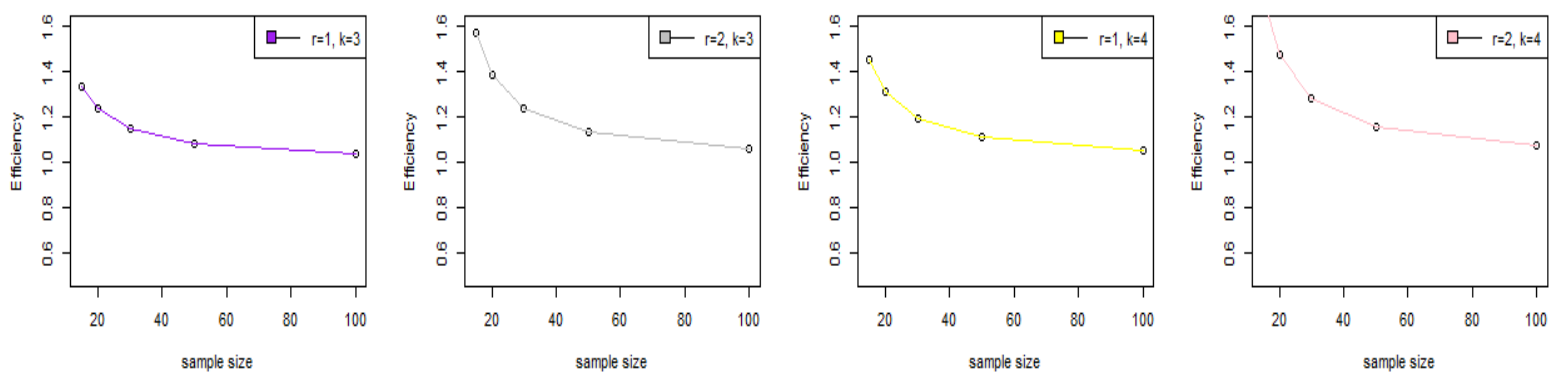

Fig. 6: Graph of Relative Efficiency of Entropy Loss Function with Respect to MLE under Extended Jeffrey's Prior for Different Values of k=1, 2, 3, 4 and $\mathrm{r}=1,2$ when $\mathrm{n}=15,20,30,50$ and 100 
Graph of R.E of entropy w.r.t. MLE using Ex Jef Graph of R.E of entropy w.r.t. MLE using Ex Jef Graph of R.E of entropy w.r.t. MLE using Ex Jef Graph of R.E of entropy w.r.t. MLE using Ex Jel
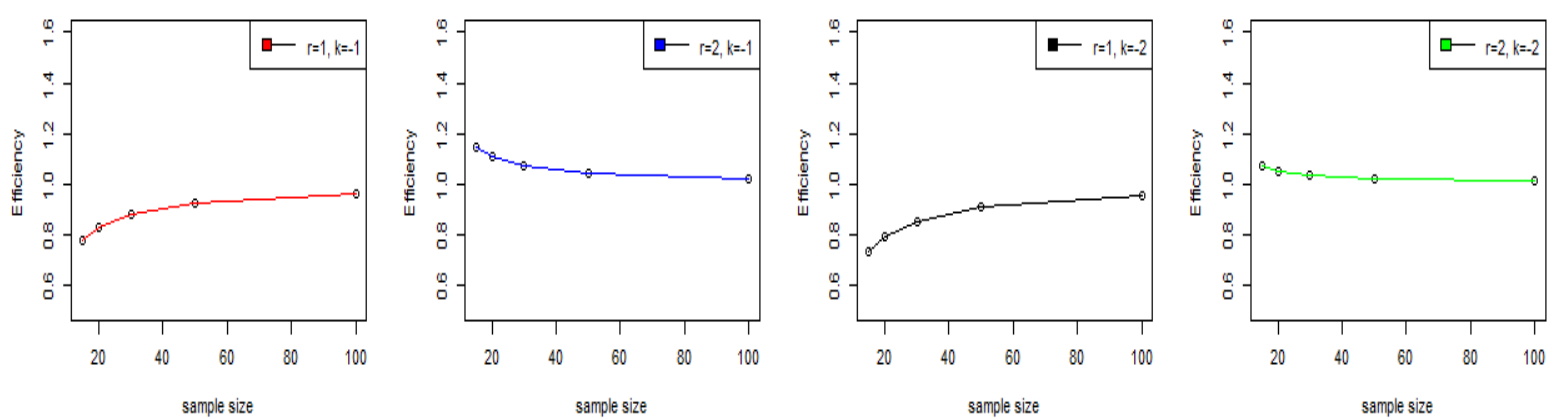

Graph of R.E of entropy w.r.t. MLE using Ex Jef Graph of R.E of entropy w.r.t. MLE using Ex Jef Graph of R.E of entropy w.r.t. MLE using Ex Jef Graph of R.E of entropy w.r.t. MLE using Ex Jel
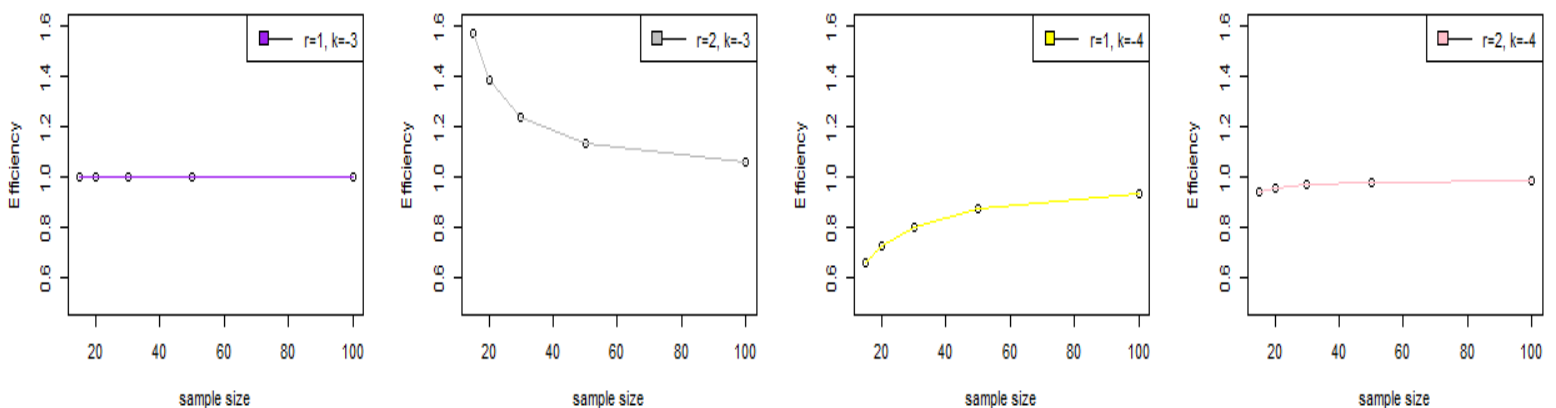

Fig. 7: Graph of Relative Efficiency of Entropy Loss Function with Respect to MLE under Extended Jeffrey's Prior for Different Values of k=-1, -2, -3, 4 and $\mathrm{r}=1,2$ when $\mathrm{n}=15,20,30,50$ and 100
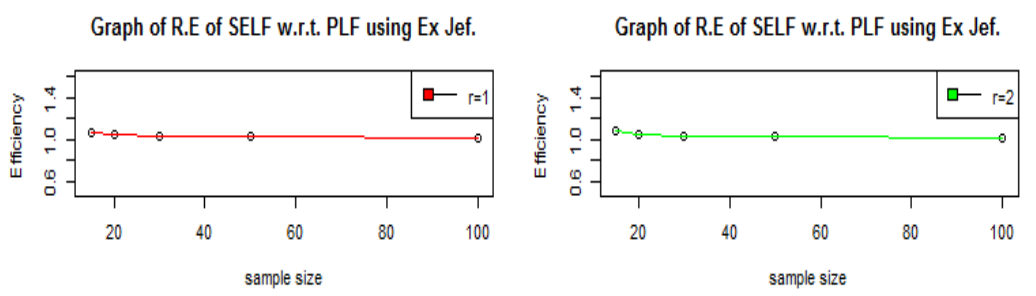

Graph of R.E of SELF w.r.t. PLF using Ex Jef.

Graph of R.E of square loss w.r.t. MLE using Ex Jef.

Graph of R.E of square loss w.r.t. MLE using Ex Jef.
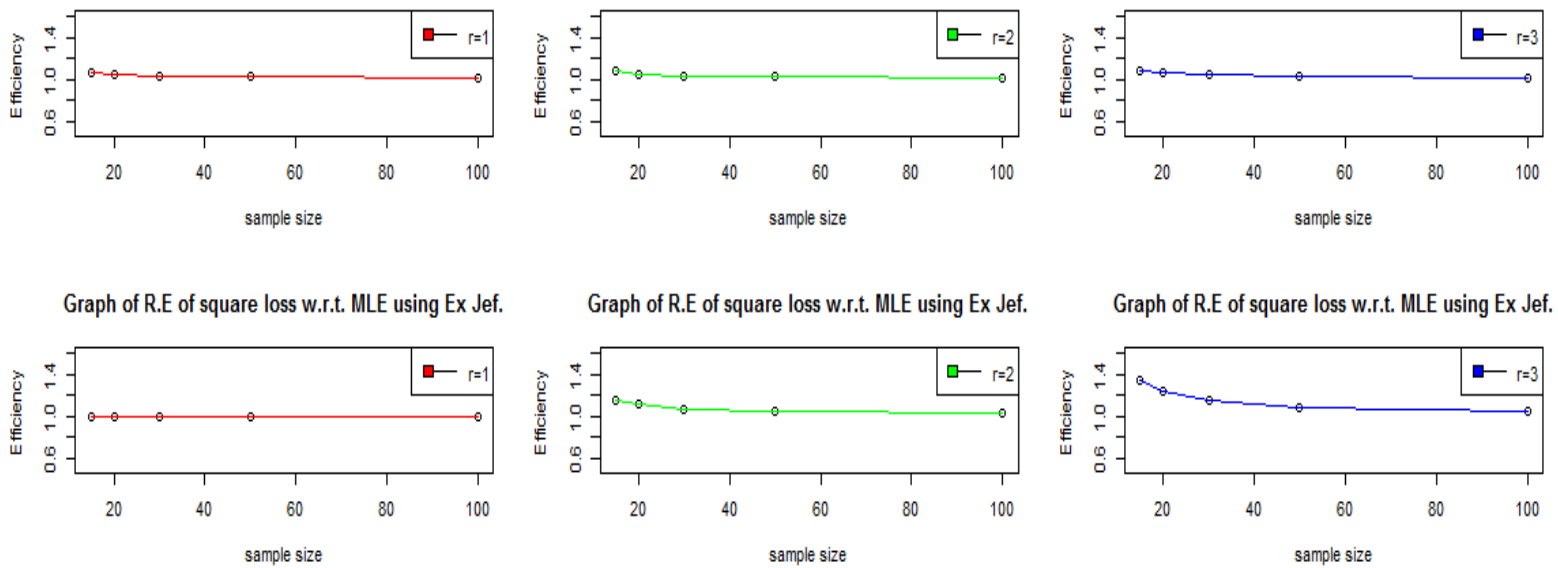

Graph of R.E of square loss w.r.t. MLE using Ex Jef.

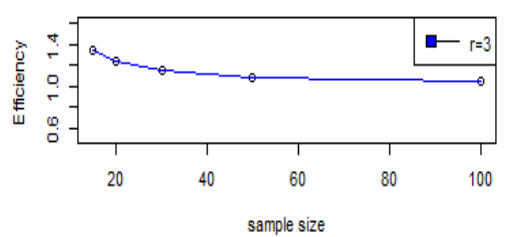

Graph of R.E of precautionary loss w.r.t. MLEusing Ex Jef. Graph ofR.E of precautionary loss w.r.t. MLE using Ex Jef. Graph of R.E of precautionary loss w.r.t. MLE using Ex Jef
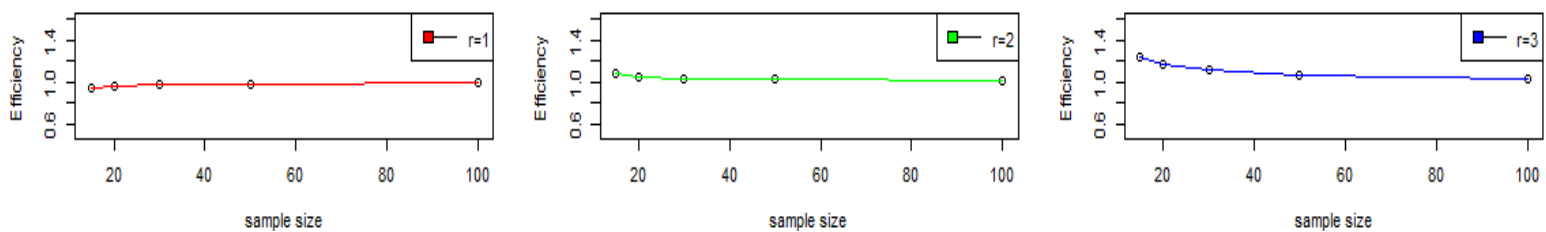

Fig. 8: Graph of Relative Efficiency of Squared Error with Respect to Precautionary, Squared Error with Respect to MLE and Precautionary Loss Function with Respect to MLE under Extended Jeffrey's Prior for Different Values of $\mathrm{k}=1,2,3,4$ and $\mathrm{r}=1,2$ when $\mathrm{n}=15$, 20, 30, 50 and 100

\subsubsection{Posterior risk}

Posterior risk under squared error loss function

$R\left(\hat{\alpha}_{\mathrm{SL}}, \alpha\right)=E\left(L\left(\hat{\alpha}_{\mathrm{SL}}, \alpha\right)\right)=E\left(\alpha^{2}\right)-(E(\alpha))^{2}$

Therefore, the posterior risk of $\hat{\alpha}_{S L}$ is given by:

$$
R\left(\hat{\alpha}_{\mathrm{SL}}, \alpha\right)=\frac{(n-r+1)}{M^{2}}
$$

The posterior risk under entropy loss function is obtained as follows:

$$
R\left(\hat{\alpha}_{E L}, \alpha\right)=E\left(L\left(\hat{\alpha}_{E L}, \alpha\right)\right)=w E\left[\left(\frac{\hat{\alpha}_{E L}}{\alpha}\right)^{k}-k \log \left(\frac{\hat{\alpha}_{E L}}{\alpha}\right)-1\right]
$$




$$
=w\left[\frac{\sqrt{n-k} \sqrt{n-r+1}}{\sqrt{n} \sqrt{n-r-k+1}}-\log \left(\frac{\sqrt{n-r+1}}{\sqrt{n-r-k+1}}\right)+k \log (n-1)-1\right]
$$

and finally, the posterior risk under the precautionary loss function is obtained as follows:

$$
R\left(\hat{\alpha}_{P L}, \alpha\right)=E\left(\hat{\alpha}_{P L}, \alpha\right)=2\left(\hat{\alpha}_{P L}-E(\alpha)\right)
$$

Therefore, the posterior risk of $\hat{\alpha}_{P L}$, is given by:

$$
R\left(\hat{\alpha}_{P L}, \alpha\right)=2\left(\frac{\sqrt{(n-r+2)(n-r+1)}}{M}-\frac{n-r+1}{M}\right)
$$

\section{Numerical comparisons}

In this section, results of some numerical experiments were obtained in order to compare the performances of the different estimators proposed in the previous section with that of MLE. Extensive Monte Carlo simulations were carried out to compare the performance of the different estimators with respect to biases and mean-squared errors (MSEs) for different sample sizes $(n=15,20$, 30,50 and 100) against different shape $(\alpha)$ parameter values of $0.25,0.5,1.0,1.5,2.0$ and 2.5. The Monte Carlo simulations were replicated 10000 times and averaged over.

Table 1:Average Estimates and Corresponding MSEs (within Parenthesis) for $n=15$ and $n=20$

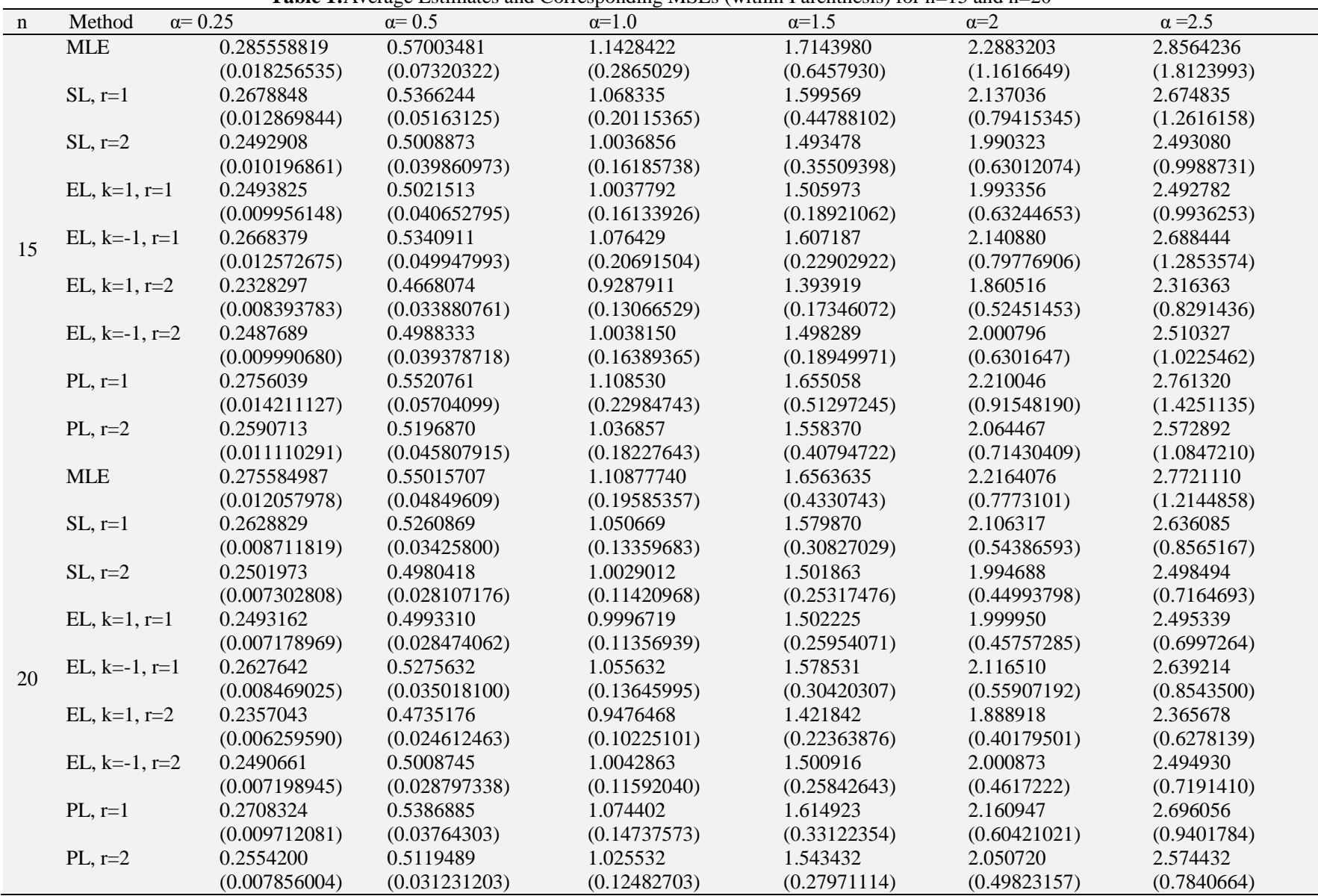

As expected, it is observed that the performances of both the Maximum likelihood and Bayesian estimates become better when sample size increases. Also the MLEs and Bayesian estimates become closer as sample size increases. The MSEs of the Bayesian estimate is

\begin{tabular}{|c|c|c|c|c|c|c|c|}
\hline $\mathrm{n}$ & Method & $\alpha=0.25$ & $\alpha=0.5$ & $\alpha=1.0$ & $\alpha=1.5$ & $\alpha=2$ & $\alpha=2.5$ \\
\hline \multirow{13}{*}{30} & \multirow[t]{2}{*}{ MLE } & 0.266518752 & 0.53514531 & 1.07019192 & 1.6018373 & 2.1322716 & 2.6624329 \\
\hline & & $(0.007174223)$ & $(0.02895296)$ & $(0.11390888)$ & $(0.2548194)$ & $(0.4523064)$ & $(0.7020738)$ \\
\hline & \multirow[t]{2}{*}{$\mathrm{SL}, \mathrm{r}=1$} & 0.2584255 & 0.5173756 & 1.038036 & 1.555971 & 2.069341 & 2.586416 \\
\hline & & $(0.005336049)$ & $(0.02023931)$ & $(0.08161683)$ & $(0.18462182)$ & $(0.32741443)$ & $(0.5110973)$ \\
\hline & \multirow[t]{2}{*}{$\mathrm{SL}, \mathrm{r}=2$} & 0.2502024 & 0.5002525 & 0.9993081 & 1.500592 & 1.993739 & 2.500098 \\
\hline & & $(0.004787001)$ & $(0.018064346)$ & $(0.07282910)$ & $(0.16506706)$ & $(0.28561993)$ & $(0.4514712)$ \\
\hline & \multirow[t]{2}{*}{$\mathrm{EL}, \mathrm{k}=1, \mathrm{r}=1$} & 0.2488482 & 0.5002077 & 0.9997663 & 1.499512 & 1.999066 & 2.508588 \\
\hline & & $(0.004664762)$ & $(0.018205672)$ & $(0.07376873)$ & $(0.16352676)$ & $(0.29164141)$ & $(0.4519725)$ \\
\hline & $\mathrm{EL}, \mathrm{k}=-1, \mathrm{r}=1$ & $(0.005181345)$ & $(0.020229817)$ & $(0.08245872)$ & $(0.18501567)$ & $(0.33010988)$ & $(0.5115743)$ \\
\hline & \multirow[t]{2}{*}{$\mathrm{EL}, \mathrm{k}=1, \mathrm{r}=2$} & 0.2407661 & 0.4834828 & 0.9681059 & 1.445357 & 1.926913 & 2.421546 \\
\hline & & $(0.004299593)$ & $(0.016648594)$ & $(0.06710460)$ & $(0.14762973)$ & $(0.26748084)$ & $(0.4106913)$ \\
\hline & \multirow[t]{2}{*}{$\mathrm{EL}, \mathrm{k}=-1, \mathrm{r}=2$} & 0.2492276 & 0.4990409 & 0.9964926 & 1.497007 & 1.998780 & 2.491784 \\
\hline & & $(0.004652067)$ & $(0.017868968)$ & $(0.07157098)$ & $(0.16182772)$ & $(0.2937815)$ & $(0.4568782)$ \\
\hline
\end{tabular}
lower than the MSEs of the MLEs. Hence, the Bayesian estimators are more stable than the MLEs.

Table 2:Average Estimates and Corresponding MSEs (within Parenthesis) for $\mathrm{n}=30,50$ and 100 


\begin{tabular}{|c|c|c|c|c|c|c|c|}
\hline & PL, $r=1$ & $\begin{array}{l}0.2622906 \\
(0.005725977)\end{array}$ & $\begin{array}{l}0.5274802 \\
(0.02173467)\end{array}$ & $\begin{array}{l}1.052114 \\
(0.08695072)\end{array}$ & $\begin{array}{l}1.582793 \\
(0.19655293)\end{array}$ & $\begin{array}{l}2.103336 \\
(0.34958272)\end{array}$ & $\begin{array}{l}2.627797 \\
(0.5478624)\end{array}$ \\
\hline & $\mathrm{PL}, \mathrm{r}=2$ & 0.2532669 & 0.5068763 & 1.017359 & 1.524587 & 2.034270 & 2.544990 \\
\hline & & $(0.004944039)$ & $(0.019277770)$ & $(0.07602797)$ & $(0.17308480)$ & $(0.30535555)$ & $(0.4842226)$ \\
\hline & MI F & 0.260090516 & 0.521780837 & 1.04228548 & 1.55944646 & 2.07742220 & 2.6014566 \\
\hline & MLE & $(0.004171391)$ & $(0.015863054)$ & $(0.06246572)$ & $(0.13935669)$ & $(0.24894747)$ & $(0.3888973)$ \\
\hline & & 0.2540292 & 0.5106458 & 1.019428 & 1.528757 & 2.036420 & 2.555916 \\
\hline & SL, r=1 & $(0.003095190)$ & $(0.01139947)$ & $(0.04522238)$ & (0.10043913) & $(0.17924122)$ & $(0.2854233)$ \\
\hline & SI $r-$ ? & 0.2487903 & 0.5005318 & 1.0016037 & 1.500484 & 2.001939 & 2.499811 \\
\hline & & 0.2497113 & 0.4998774 & 1.0013999 & 1.500827 & 2.000067 & 2.497600 \\
\hline & $\mathrm{EL}, \mathrm{K}=1, \mathrm{r}=1$ & $(0.002857177)$ & $(0.010424658)$ & $(0.04187141)$ & (0.09480684) & $(0.16783165)$ & $(0.2637163)$ \\
\hline & & 0.2548652 & 0.5100395 & 1.022284 & 1.535469 & 2.039084 & 2.555111 \\
\hline 50 & $\mathrm{EL}, \mathrm{k}=-1, \mathrm{r}=1$ & $(0.003034190)$ & $(0.011293011)$ & $(0.04522616)$ & $(0.10352546)$ & $(0.18070001)$ & $(0.2833150)$ \\
\hline & FI $\mathrm{k}-1 \mathrm{r}-$ ? & 0.2442470 & 0.4882093 & 0.9790828 & 1.470826 & 1.955461 & 2.448781 \\
\hline & $\mathrm{EL}, \mathrm{K}=1, \mathrm{r}=2$ & $(0.002710622)$ & $(0.009890655)$ & $(0.04026443)$ & (0.08960196) & $(0.16044452)$ & $(0.2514753)$ \\
\hline & & 0.2495269 & 0.5012636 & 1.0021887 & 1.499406 & 1.997564 & 2.498958 \\
\hline & $\mathrm{EL}, \mathrm{k}=-1, \mathrm{r}=2$ & $(0.002827207)$ & $(0.010526118)$ & $(0.04273875)$ & $(0.09339190)$ & $(0.1700191)$ & $(0.2611444)$ \\
\hline & PL, r=l & $(0.003173853)$ & $(0.01182771)$ & $(0.04745128)$ & $(0.10594323)$ & $0.19040767)$ & $(0.2929627)$ \\
\hline & & 0.2511433 & 0.5048657 & 1.011027 & 1.517659 & 2.020152 & 2.533191 \\
\hline & $\mathrm{PL}, \mathrm{r}=2$ & $(0.002975853)$ & $(0.010793183)$ & $(0.04391043)$ & $(0.09817375)$ & $(0.17343730)$ & $(0.2752971)$ \\
\hline & MI F & 0.252916501 & 0.509738443 & 1.01985270 & 1.53025829 & 2.03867602 & 2.55020387 \\
\hline & MILE & $(0.002443436)$ & $(0.007234328)$ & $(0.02881461)$ & $(0.06510115)$ & $(0.11608332)$ & $(0.18134812)$ \\
\hline & & 0.2503591 & 0.5049108 & 1.010304 & 1.513652 & 2.022072 & 2.528728 \\
\hline & $\mathrm{SL}, \mathrm{r}=1$ & $(0.001848853)$ & $(0.00526002)$ & $(0.02130580)$ & (0.04797307) & $(0.08485918)$ & $(0.1343140)$ \\
\hline & & 0.2478385 & 0.4996020 & 1.0011630 & 1.500530 & 2.002233 & 2.496723 \\
\hline & SL, r=2 & $(0.001859602)$ & $(0.005132773)$ & $(0.02066969)$ & $(0.04588683)$ & $(0.08227402)$ & $(0.1266430)$ \\
\hline & FI $k-1 \quad r-1$ & 0.2483971 & 0.5004839 & 0.9989757 & 1.499899 & 2.001247 & 2.500503 \\
\hline & $E L, K=1, r=1$ & $(0.001738842)$ & $(0.005169403)$ & $(0.02051745)$ & $(0.04598710)$ & $(0.08233682)$ & $(0.1285524)$ \\
\hline & & 0.2507855 & 0.5048234 & 1.010069 & 1.514847 & 2.020954 & 2.528189 \\
\hline 100 & $\mathrm{EL}, \mathrm{k}=-1, \mathrm{r}=1$ & $(0.001824803)$ & $(0.005349108)$ & $(0.02135144)$ & $(0.04812344)$ & $(0.08526826)$ & $(0.1339325)$ \\
\hline & & 0.2453083 & 0.4946687 & 0.9901949 & 1.482126 & 1.980570 & 2.470485 \\
\hline & $\mathrm{EL}, \mathrm{k}=1, \mathrm{r}=2$ & $(0.001821130)$ & $(0.004990276)$ & $(0.02006835)$ & $(0.04488398)$ & $(0.08039079)$ & $(0.1261188)$ \\
\hline & FI $k-1, r-2$ & 0.2479325 & 0.5000948 & 0.9979656 & 1.499204 & 1.999122 & 2.503251 \\
\hline & $-1, r=2$ & (0.001812998) & $(0.005137149)$ & (0.02010039) & $(0.04587503)$ & $(0.0816968)$ & $(0.1285189)$ \\
\hline & & 0.2493552 & 0.5031723 & 1.004760 & 1.509310 & 2.007441 & 2.513429 \\
\hline & PL, r=2 & $(0.001841481)$ & $(0.005228682)$ & $(0.02065068)$ & $(0.04729740)$ & $(0.08233816)$ & $(0.1299110)$ \\
\hline
\end{tabular}

The estimates under the extended Jeffrey's prior is more stable than the MLEs since the MSEs under the extended Jeffrey's prior is smaller than the MSEs. under MLEs. Under the extended Jeffrey's prior, the MSEs when $r=2$ were found to be smaller than the MSEs when $r=1$. Hence, the estimates are more stable when $r=2$ than when $\mathrm{r}=1$.

It is further observed that the Bayesian estimators under entropy and squared error loss functions perform better than the Bayesian estimates under the precautionary loss function and the estimates under the entropy loss function have a lower mean square error than the estimates under the squared error loss function.

Figs. 2-8 shows the Relative Efficiency of estimators for different values of $n, r$ and $k$. The graphs provide an impression on how the efficiencies change with changes in the values of $n, r$ and $k$. The graphs are plotted using efficiency against sample sizes. It is observed from Figs. 2, 4 and 6 that as sample size increases, the efficiency decreases while the efficiencies increase when $\mathrm{k} \leq-2$ in Fig. 3 and in Fig 5 when $\mathrm{k} \leq-3$. In Fig. 7, the efficiencies fluctuate when $\mathrm{k}$ is between -3 and -1 but increase when $\mathrm{k} \leq-4$. It is further observed that the rate of decrease/ increase in these graphs varies for different values of $\mathrm{k}$ and as sample size becomes very large, the effect of $k$ on efficiency is negligible.

Fig. 8 reveals the relative efficiency of $\widehat{\alpha}_{S L}$ with respect to $\widehat{\alpha}_{P L}$ of $\alpha, \widehat{\alpha}_{S L}$ with respect to $\widehat{\alpha}_{\text {MLE }}$ of $\alpha$ and $\widehat{\alpha}_{\mathrm{PL}}$ with respect to $\widehat{\alpha}_{\mathrm{MLE}}$ of $\alpha$. It is observed that the relative efficiency of $\widehat{\alpha}_{\mathrm{SL}}$ with respect to $\widehat{\alpha}_{\mathrm{PL}}$ of $\alpha$ is always greater than one when $r \geq 1$ and decreases when sample size is large. The relative efficiency of $\widehat{\alpha}_{S L}$ with respect to $\widehat{\alpha}_{M L E}$ of $\alpha$ and that of $\widehat{\alpha}_{P L}$ with respect to $\widehat{\alpha}_{M L E}$ of $\alpha$ are greater than one when $r \geq 2$. When $r=1$, the relative efficiency of $\widehat{\alpha}_{S L}$ with respect to $\widehat{\alpha}_{M L E}$ of $\alpha$ is equal to one and is less than one for the relative efficiency of $\widehat{\alpha}_{\mathrm{PL}}$ with respect to $\widehat{\alpha}_{\mathrm{MLE}}$ of $\alpha$.

\section{Conclusion}

In this paper, Bayes estimation of the shape parameter of generalized Rayleigh distribution is considered. Non-informative prior is assumed under the assumption of squared error, entropy and precautionary los functions. Comparison between the performances of the MLEs and the Bayes estimators were carried out and it was observed that Bayes estimator under the Entropy loss function is better than the Bayes estimators under the squared error and precautionary loss functions and that of MLEs.

\section{References}

[1] Abdel-Hady, D. H. "Bivariate Generalized Rayleigh Distribution." Journal of Applied Sciences Research, no. 9 (2013): 5403-5411.

[2] Al-Kutubi, H.S. On comparison of estimation procedures for parameter and survival function exponential distribution using simulation, Ph.D. Thesis, College of Ibn Al-Hatham. Baghdad University, (2005).

[3] Burr, I. W. "Cumulative Frequency Distribution." Annual of Mathematical Statistics $13 \quad$ (1942): $215-232$ http://dx.doi.org/10.1214/aoms/1177731607.

[4] Calabria, R., and Pulcini, G.. "An engineering approach to Bayes estimation for the Weibull distribution ." Micro-electron. Reliab. 34 , no. 5 (1994): 789-802. http://dx.doi.org/10.1016/00262714(94)90004-3.

[5] Dey, D. K., Gosh M., and Srinivasan C.. "Simultaneous estimation of parameters under entropy loss." Journal of Statist. Plan. and Infer., (1987): 347-363.

[6] Dey, D. K., and Liu Pei-San L.. "On comparison of estimators in a generalized life model." Micro-electron. Reliab. 32, no. 1 (1992): 207-221. http://dx.doi.org/10.1016/0026-2714(92)90099-7.

[7] Jeffreys, H. Theory of Probability. Oxford, Clarendon Press, 1961.

[8] Kundu, D., and Raqab M. Z.. "Generalized Rayleigh distribution: different methods of estimations." Computational Statistics \& Data 

Analysis
$49 \quad$ (2005):
187
200.

http://dx.doi.org/10.1016/j.csda.2004.05.008.

[9] Lio, Y. L., Chen, D. and Tsai, T. "Parameter Estimations for Generalized Rayleigh Distribution under Progressively Type-I Interval Censored Data." American Open Journal of Statistics, (2011): 46-57.

[10] Mahdi, S. "Improved Parameter Estimation in Rayleigh Model." Metodološki zvezki 3, no. 1 (2006): 63-74.

[11] Norstrom, J. G. "The use of precautionary loss functions in risk analysis." IEEE Trans. Reliab. 45, no. 3 (1996): 400-403. http://dx.doi.org/10.1109/24.536992.

[12] Raqab, M.Z. and Kundu D. "Comp arison of Different Estimators of $\mathrm{P}[\mathrm{Y}<\mathrm{X}]$ for a Scaled Burr Type X Distribution." Commun. Statist. $\quad$-Simul. $\quad$ Comp. $34 \quad$ (2005): 465 - 483. http://dx.doi.org/10.1081/SAC-200055741.

[13] Samaila, M., and Cenac M. "Estimating and Assessing the parameters of the logistic and Rayleigh distribution from three methods of estimation." Carrib. J. Math. comput. sci 13 (2006): 25 34.

[14] Surles, J.G., and Padgett W.J.. "Inference for reliability and stressstrength for a scaled Burr Type X distribution." Lifetime Data Anal. 7, (2001): 187-200. http://dx.doi.org/10.1023/A:1011352923990.

[15] Surles, J.G., and Padgett W.J.. "Some properties of a scaled Burr type X distribution." J. Statist. Plann. Inference, (2004). 